\title{
Programa de Promoção da Inteligência Emocional na Ansiedade Infantil
}

\section{Programme for Promotion of Emotional Intelligence in Childhood Anxiety}

\author{
Marina Sousa*, Joana Benevides*, Célia Barreto Carvalho** \& Suzana Nunes Caldeira*** \\ * Universidade dos Açores, Portugal \\ ** Universidade dos Açores e Centro de Investigação do Núcleo de Estudos e Intervenção Cognitivo-Comportamental, Portugal \\ ***Universidade dos Açores - CICS.NOVA.UAçores, Portugal
}

\begin{abstract}
Resumen
A Inteligência Emocional (IE) é um dos componentes mais importantes para o ajustamento psicológico das crianças. Há evidência de que as crianças com perturbações de ansiedade apresentam défices na IE, pelo que se pretende desenvolver um programa de intervenção de cariz remediativo para a promoção da IE junto de crianças com ansiedade patológica. Espera-se que este programa possa contribuir para o delineamento de intervenções mais adequadas e direcionadas às necessidades destas crianças, permitindo reduzir os custos associados às intervenções na área da saúde e da educação e que possa auxiliar os profissionais a intervir na área da saúde mental infanto-juvenil.

Palavras chave: neuroses e perturbações afectivas, terapia familiar e de grupo, educação especial e remediativa.
\end{abstract}

\begin{abstract}
Emotional intelligence (EI) is one of the most important components for the psychological adjustment of children. There is evidence that children with anxiety disorders present deficits in EI, so we want to develop a intervention program of healing nature for the promotion of EI with children with pathological anxiety. It is expected that this program will contribute to the design of more appropriate interventions and targeted to the needs of these children, reducing the costs associated with the interventions in health and education and that may assist professionals to intervene in the child and youth mental health field.

Keywords: neuroses \& affective disorders, group \& family therapy, special \& remedial education.
\end{abstract}

\section{Introdução}

A Inteligência Emocional (IE) é um dos componentes mais importantes para o ajustamento psicológico do indivíduo e, em particular, das crianças. A IE “envolve a habilidade de perceber com precisão, avaliar $e$ expressar a emoção; a capacidade de acessar e/ou gerar sentimentos facilitadores do pensamento, a capacidade de compreender a emoção e conhecimento emocional; $e$ a capacidade de regular as emoções para promover o crescimento emocional e intelectual" (Mayer \& Salovey, 1997, p. 10). Para Mayer e Salovey (1997), a IE é então caracterizada pela capacidade de expressar, reconhecer, identificar, diferenciar, compreender, avaliar, gerir e regular adequadamente as emoções em si e nos outros, com vista à promoção do crescimento emocional e intelectual do indivíduo. De acordo com o Modelo da IE desenvolvido por Mayer e Salovey (1997) existem 4 grandes grupos de habilidades que são desenvolvidas de forma gradual e crescente de complexidade: 1) Capacidade de perceber emoções, que inclui a percepção, avaliação e expressão emocional; 2) Emoções facilitadoras do pensamento; 3 ) Compreensão e análise das emoções e 4) Regulação reflexiva das emoções (gerir emoções) (Mayer \& Salovey, 1997).

A primeira categoria (percepção, avaliação e expressão emocional), respeita à precisão com que os sujeitos são capazes de identificar emoções (Mayer \& Salovey, 1997). A segunda categoria está relacionada com a capacidade das emoções ajudarem os sujeitos a pensar. Consideram-se os eventos emocionais facilitadores do pensamento, isto é, a emoção é um sistema que permite ao sujeito sinalizar mudanças importantes, que tanto podem ocorrer na pessoa, como no ambiente envolvente.

Para além disto, é possível antecipar emoções para facilitar a tomada de decisão ou auxiliar no processo de planeamento. A terceira categoria de habilidades (compreensão e análise de emoções) implica compreender e analisar emoções, bem como antecipar os trajectos prováveis no tempo e compreender os seus resultados. A criança ao reconhecer emoções, irá rotulá-las e tentar perceber que relações existem entre elas. Para além do reconhecimento de semelhanças e diferenças entre emoções, também surgem emoções contraditórias e combinações de emoções. Por fim, a quarta categoría, a regulação emocional, diz respeito à capacidade de tolerar e gerir sentimentos. Nesta fase inicia-se a divisão entre emoções e comportamentos e as crianças começam a perceber que estas duas componentes (comportamento e emoção) podem estar separadas, ao mesmo tempo que aprendem diferentes estratégias de auto-regulação emocional que auxiliam a criança a responder às situações que se lhe impõe (Mayer \& Salovey, 1997).

Sendo o construto de IE relativamente recente, o que é facto é que as emoções acompanharam a evolução das espécies, em particular a humana, mostrando-se indispensáveis à sua sobrevivência (e.g. Dixon, 1998; Gilbert, 2000). As emoções surgem em resposta a um acontecimento que, ao ser interno ou exterior ao indivíduo, tem um sentido positivo ou negativo para si. 
Por outras palavras, estando os acontecimentos quotidianos envoltos de informação afectiva, é o modo como esta informação afectiva é processada que varia de indivíduo para indivíduo, de acordo com as suas habilidades emocionais (Mayer \& Salovey, 1990). Quer isto dizer que, apesar de existirem diferenças individuais em termos de estilos de processamento e habilidades que culminam em diferenças na capacidade de compreender, expressar e gerir emoções, tais habilidades podem ser aprendidas e contribuir para a saúde mental dos indivíduos (Mayer \& Salovey, 1997).

Foi ao longo desta evolução, que o ser humano e as restantes espécies foram desenvolvendo e aperfeiçoando determinadas estratégias de defesa contra possíveis ameaças sociais que pudessem comprometer a sua sobrevivência, como é o caso das estratégias de luta ou fuga (Dixon, 1988; Gilbert, 2008). O ser humano, à semelhança do que acontece com os outros animais, recorre a estratégias de luta quando avalia que a situação ameçadora pressupõe uma competição por recursos básicos (e.g. alimentação, reprodução) e a manutenção da posição ocupada numa determinada hierarquia social (dominante) (Dixon, 1998; Gilbert, 1998). Nestas situações, o adversário é avaliado como sendo mais fraco, pelo que a estratégia de luta assume maior eficácia (Dixon, 1998). Em contrapartida, as estratégias de fuga envolvem o evitamento/afastamento da situação considerada ameaçadora ou passível de causar dano, o que permite garantir a integridade individual e social do indivíduo (Dixon, 1998). Aqui, o adversário é avaliado como sendo mais forte, por isso a fuga da situação é o que melhor garante a sobrevivência (Dixon, 1998).

A perspectiva evolucionária permite uma complementaridade entre a evolução que ocorreu nas espécies e no ser humano com os sinais emocionais e sociais que se apresentam em situações de sobrevivência. Destaca-se que estes comportamentos de sobrevivência encontram-se hoje patentes em muitas das situações ansiógenas manifestadas pelas pessoas. Por exemplo, quando o ser humano se defronta com uma situação reconhecida como perigosa, tende a desenvolver sinais de ansiedade, contraindo a sua postura corporal, com vista a preparar o organismo para fugir da situação ou para lutar (e.g. comportamentos biologicamente determinados que se encontram presentes também nos outros animais) (Gilbert, 2000; Carvalho, 2009).

Esta noção de evolução das emoções e de determinadas estratégias/habilidades emocionais com vista à sobrevivencia da espécie foi também apresentada por Ekman (1994). Segundo o autor, durante a evolução das espécies, o ser humano desenvolveu estratégias adaptativas em situações avaliadas como ameaçadoras e capazes de ativar determinadas respostas fisológicas e comportamentais próprias de cada emoção e indispensáveis à sobrevivencia da espécie (Ekman, 2003). Disto resultou o modelo das emoções básicas desenvolvido por Ekman (2003), no qual se destaca a universalidade de determinadas das emoções (e.g. alegria, tristeza, o medo, raiva, surpresa, nojo).

Ekman (2003) destaca que de facto não temos controlo sobre aquilo em que nos tornamos emocionalmente, mas podemos realizar pequenas mudanças na forma como as nossas emoções são activadas e no modo como nos comportamos emocionalmente.

Como já foi sendo mencionado anteriormente, sendo a vantagem das emoções a sobrevivencia da espécie, para Ekman (2003) são elas que permitem ao indivíduo ativar, de forma rápida, planos de acção para fazer face às situações que decorrem no quotidinao do indivíduo. Portanto, as emoções possuem mecanismos automáticos de avaliação que permitem ao ser humano monitorizar de forma continuada o ambiente à sua volta e detectar quando uma ameaça ao bem-estar ou à sobrevivência pode ocorrer (Ekman, 2003).

Durante a infância a ansiedade surge como uma experiência transitória e funcional capaz de facilitar a adaptação a situações novas, inesperadas ou perigosas (Rosen \& Schulkin, 1998). Alguns autores referem que é possível que crianças que tenham experenciado ansiedade na infância, tenham maior probabilidade de desenvolver perturbações depressivas ou outras pataologias psiquiátricas na vida adulta, capazes de influenciar o desenvolvimento académico, familiar, social e emocional (Gonçalves \& Hedt, 2009; Wilson, Pritchard, \& Revalee, 2005).

De um modo geral, podemos dizer que as crianças com ansiedade possuem preocupações ou medos exagerados com a saúde ou desempenho em testes, agressão física, problemas com os pares, hipersensibilidade aos sinais de perigo, comportamentos de evitamento, entre outros (Bernstein \& Borchardt, 1991; Layne, Bernart, Victos \& Bernstein, 2008; Pina, Silverman, Alfano \& Saavedra, 2002; Caíres \& Shinohara, 2010). Os sintomas ansiógenos possuem consequências negativas para a criança, nomeadamente prejudicam a sua autonomia e auto-estima o seu desempenho escolar, as suas interacções sociais, aumentando o isolameto social em virtude dos menores níveis de aceitação e suporte por parte do grupo de pares, que por sua vez aumenta a probabilidade destas crianças serem ignoradas, rejeitadas ou ridicularizadas. Para além disso, níveis de ansiedade elevados em testes e outras atividades escolares resultam na diminuição do rendimento académico e até numa recusa em ir à escola (Beidel, Turner \& Morris, 1999; Caíres \& Shinohara, 2010; Castillo, Recondo, Asbahr \& Manfro, 2000; Filho \& Silva, 2013; Janeiro, 2013; Vianna, Campos \& Landeira-Fernandez, 2009).

Embora haja escassez de estudos de prevalência das doenças mentais infanto-juvenis no nosso país, sabe-se que Portugal é um dos países da Europa com os índices mais elevados de doença mental (23\%), sendo a mais comum a ansiedade (16.5\%) (Direcção Geral da Saúde, 2013). A par deste indicador, a investigação aponta para uma correlação significativa entre a ansiedade, em particular a de desempenho, com a insatisfação com a escola, com as reprovações académicas (e.g. Janeiro, 2013) e, consequentemente, com os indíces de insucesso escolar que, em Portugal, se situam nos 25\% (Justino, Pascoeiro, Franco, Santos, Almeida \& Baptista, 2014).

Devido à gravidade crescente desta problemática, a intervenção precoce que passe pela implementação de programas de promoção da IE torna-se premente, de forma a diminuir os índices de problemas de saúde mental e escolares na infância. Neste âmbito destacam-se 
os programas SEL (Social and Emotional Learning, 2013), validados empiricamente, com eficácia não só ao nível de melhorias na IE, como na redução de sintomatologia ansiógena (SEL, 2013). Um estudo piloto desenvolvido em escolas do $1^{\circ}$ e $3^{\circ}$ ciclos da Ilha de São Miguel demonstrou efeitos positivos ao nível do conhecimento e identificação de emoções básicas e secundárias (em si e no outro), da aquisição de competências de regulação emocional, da empatia e da assertividade, desenvolvendo-se mecanismos protetores cruciais à prevenção de comportamentos de risco, de problemas de saúde mental e para o insucesso escolar nas vertentes do rendimento e do comportamento (Amaral, 2014).

A relação entre a IE e variáveis emocionais, como a ansiedade, é uma área de investigação recente, bem como do impacto desta relação no ajustamento interpessoal da criança (Aminabadi, Pourkazemi, Babapour \& Oskouei, 2012; Extremera \& Pizarro, 2006; Jacobs, et al., 2008; Mara \& Mara, 2010; Ruiz-Aranda, Salguero \& Cabello, 2012; Shamsy, Khoshli \& Asadi, 2013). No entanto, há já evidência de que as crianças com perturbações de ansiedade (e.g. ansiedade social e generalizada) apresentam défices na IE, ou seja, dificuldades em perceber, usar, compreender e regular as emoções em si e nos outros (Aminabadi et al., 2012; Extremera \& Pizarro, 2006; Jacobs et al., 2008; Mara \& Mara, 2010; Ruiz-Aranda et al., 2012; Shamsy et al., 2013) A IE parece então ser um factor de vulnerabilidade para o desenvolvimento de distúrbios de ansiedade. A investigação crescente no campo das emoções tem sugerido que as crianças com ansiedade sofrem de maior reatividade emocional e de défices na regulação emocional (Hannesdottir \& llendick, 2007; Mennin, Holaway, Fresco, Moore \& Heimberg, 2007). Um estudo realizado por Suveg e Zeman, (2004) relatou que as crianças com ansiedade têm maior reatividade emocional quando comparadas com as crianças do grupo de controlo. Nesse estudo evidencia-se que as crianças do grupo experimental são menos eficazes em aplicar estratégias de regulação emocional e na diferenciação das emoções (Mennin et al, 2005; Salters-Pedneault et al., 2006; Turk et al., 2005), manifestando igualmente uma expressão desregulada de emoções, como a raiva e a tristeza (Suveg \& Zeman, 2004). Note-se que a percepção das competências emocionais tem estado positivamente associadas com a saúde mental e ajustamento psicológico na população não-clínica (Martins, Ramalho \& Morin, 2010). Assim, podemos concluir que a elevada percepção de competências emocionais prepara os sujeitos para os desafios da vida e para a regulação emocional. Todavia, esta percepção de competências emocionais também se encontra associada com os sintomas clínicos. Especificamente, os ansiógenos possuem maior consciência das suas emoções, porém falta-lhes as estratégias de regulação emocional (Lizeretti, Extremera \& Rodríguez, 2012).

Considera-se que é durante a infância e a adolescência que o crescimento individual e, em particular, o emocional, assume uma maior importância, realçando a importância de intevenções cada vez mais precoces ao nível da promoção da IE (Cole, Michel \& Teti, 1994).
Numa revisão sistemática de literatura levada a cabo por Gonçalves e Heldt (2009) verificou-se que todos os trabalhos incluidos (embora de diferentes países) evidenciaram que os transtornos de ansiedade na infância e mesmo apenas a presença de sintomas ansiógenos (sem transtorno diagnosticado) são preditores e podem actuar como factores de risco para o desenvolvimento de psicopatologia na vida adulta, tal como mencionado anteriormente.

Estas ideias e dados expostos justificam que se deva avançar para o desenvolvimento de um programa remediativo que verse sobre a promoção da IE em crianças com ansiedade patológica, dada a necessidade já meniconada de intervenções cada vez mais precoces na saúde mental infanil e juvenil, para que seja possível prevenir patologías do foro psiquiátrico na vida adulta ou minimizar a gravidade e o impacto da sintomatología já evidenciada (Gonçalves \& Heldt, 2009).

\section{Método}

\section{Objectivos}

O programa que está em desenho possui como objectivo geral promover o desenvolvimento da inteligência emocional em crianças, de modo a reduzir a sua ansiedade e a facilitar o seu ajustamento psicológico.

Como objectivos específicos visa: a) desenvolver o conhecimento acerca das emoções; b) promover a capacidade de reconhecer emoções em si e nos outros; c) explorar formas de lidar com as diferentes emoções; d) destacar formas adequadas de lidar com as emoções; e) impulsionar o papel activo da criança para a sua regulação emocional; f) treinar competências emocionais e sociais no contexto específico da ansiedade patológica.

De forma a dar resposta a estes objetivos específicos, o programa será organizado por um conjunto de sessões, organizadas por módulos, que se prevêm incidir sobre temáticas como as emoções básicas (medo, alegria, tristeza, surpresa, nojo, raiva), as emoções sociais (vergonha, culpa) e as competências sociais (assertividade, empatia).

\section{Participantes}

Prevê-se que os participantes do estudo sejam crianças de ambos os sexos, com idades compreendidas entre os 6 e os 10 anos com diagnóstico de ansiedade efetuado pelo seu pediatra ou médico asistente. Serão excluídos do estudo crianças com deficiência mental, necessidades educativas especiais e/ou dificuldades de aprendizagem.

\section{Instrumentos}

A avaliação será realizada por escalas de auto-relato, de cariz quantitativo, tais como: o CBCL (Child Behavior Check List), elaborado por Achenbach (1991) e adaptado para a população portuguesa por Fonseca, Simões, Rebelo, Ferreira e Cardoso (1994), o Mini-Exame do Estado Mental (MMSE), elaborado por Tinetii, Richman e Powellv (1990), sendo utilizada a versão portuguesa aferida por Guerreiro, Silva, Botelho, Leitão, Caldas e Garcia, (1994); a EADS-C (Escala de depressão, ansiedade e stresse para crianças) elaborada por Lovibond e Lovibond (1995) validada para a 
população portuguesa por Pais, Ribeiro, Honrado e Leal (2004); a RCMAS (Revised Children's Manifest Anxiety Scale) de Reynolds e Richmond (1994) e adaptada para a população portuguesa por Martins (2000); o QIEC (Questionário de Inteligência Emocional para Crianças) de Barreto Carvalho e Caldeira (2014), o ERE (Escala de Regulação Emocional) de Barreto Carvalho e Martins (2014); e a EPM (Escala de Percepção do Medo) de Freitas-Magalhães e Batista (2009). De modo a adicionar informação aos dados quantitativos, pretende-se recorrer à análise de unidades de registo com recurso a diários de bordo. Com o avanço do conhecimento do conhecimento na área, esta avaliação pode vir a sofrer ajustamentos.

\section{Procedimentos de recolha de dados}

Os procedimentos envolvidos no estudo terão em conta os cuidados éticos da declaração de Elsínquia, da legislação Europeia e do código deontológico da Ordem dos Psicólogos Portugueses e obter aprovação da comissão nacional de protecção de dados (CNPD). Aquando das aprovações éticas que se prevêm solicitar às Unidades de Saúde e Unidade Hospitalar da ilha de São Miguel, proceder-se-á à explicação dos objectivos e conteúdos do programa, sendo garantida a confidencialidade e anonimato da informação nrecolhida por parte dos participantes. Estes poderão desistir da investigação a qualquer momento, estando a sua participação sujeita a consentimento informado a ser autorizada pelos pais ou representantes legais da criança.

$\mathrm{Na}$ fase de implementação do programa, prevê-se que as amostras de crianças com ansiedade patológica sejam aleatorizadas nas condições experimental e controlo. Ambos os grupos serão sujeitos às mesmas avaliações pré-teste, pós-teste e follow-up, com vista a verificar possíveis diferenças nos resultados obtidos entre os dois grupos ao nível das variáveis que se pretendem medir no estudo.

\section{Procedimentos de análise de dados}

No tratamento estatístico dos dados prevé-se recorrer à versão 21.0 do Softaware de Análise Estatística Statistical Packadge for Social Sciences (SPSS) e à metodología de análise de conteúdo.

\section{Resultados Esperados}

Aspira-se a que o desenvolvimento deste programa possa ser um contributo para os profissionais a intervir na área da saúde mental e educação infantil e juvenil, através do delineamento de intervenções precoces ou programas de reabilitação mais adequados e direcionados às necessidades destas crianças, permiteindo reduzir os custos associados às intervenções terceárias na área da saúde e da educação.

\section{Referências}

Amaral, S. (2014). Promovendo a regulação emocional em crianças do $1^{\circ}$ ciclo do Ensino Básico: Um programa de competências emocionais e socias. Tese de mestrado em Psicologia da Educação, especialidade em contextos educativos. Univeridade dos Açores.

Aminabadi, N-A., Pourkazemi, M., Babapour, J., \& Oskouei, S-G. (2012). The impact of maternal emotional intelligence and parenting style on child anxiety and behavior in the dental setting. Medicina oral, patología oral y cirurgia bucal, 17(6), 1089-1095. doi: 10.4317/medoral.17839.

Beidel, D. C., Turner, S. M., \& Morris, T. L. (1999). Psychopathology of childhood social phobia. Journal of the American Academy of Child and Adolescent Psychiatry, 38, 643-650.

Borges, A., I., Manso, D., S., Tomé, G., \& Matos, M., G. (2008). Ansiedade e coping em crianças e adolescentes: Diferenças relacionais com a idade $e$ género, 4(26), 551-561.

Caíres, M., C., \& Shinohara, H. (2010). Trantornos de ansiedade na criança: Um olhar nas comunidades. Revista Brasileira de Terapia Cognitivas, 1(6), 62-84.

Carthy, T., Horesh, N., Apter, A., Edge, M.D., \& Gross, J. (2010). Emotional reactivity and cognitive regulation in anxious children. Behaviour research and therapy, 48(5), 384-93. doi: 10.1016/j.brat.2009.12.013.

Carvalho, C. (2009). Psicose Esquizofrénica. Crenças Paranóides: exploração da sua Etiologia na população normal e em doentes com esquizofrenia. Tese de Doutoramento em Psicologia Clínica. Faculddae de Psicologia e de Ciências da Educação da Universidade de Coimbra.

Castillo, A., R., G., L., Recondo, R., Asbahr, F., R., \& Manfro, G., G. (2000). Transtornos de ansiedade. Revista Brasileira de Psiquiatria. 22(2), 20-23.

Collaborative for Academic, Social and Emotional Learning (2013). Effetive Social and Emotional Learning Programs, preschool and elementary School Edition. Chicago: CASEL. Disponível em: http://www.casel.org/research.

Direcção Geral da Saúde (DGS, 2014). Portugal: Saúde Mental em números-2014; DGS: Lisboa, Portugal. Disponível em: http://www.dgs.pt/?cr=26728.

Dixon, A. K. (1998). Ethological strategies for defence in animals and humans: Their role in some psychiatric disorders. British Journal of Medical Psychology, 71, 417-445.

Ekman, P. (2003). Emotions revealed: Recognizing faces and feelings to improve communication and emotional life. New York: Time Books.

Extremera, N., \& Pizaro, D. (2006). The Role of Emotional Intelligence in Anxiety and Depression among Adolescents. Individual Differences Research, 4(1), 16-27.

Filho, O., C., S., \& Silva, M., P. (2013). Transtornos de ansiedade em adoelscentes: Considerações para a pediatria e hebiatria. Revista Adolescência e Saúde. 3(10), 31-41.

Gilbert, P. (1998). Evolutionary psychopathology: Why isn't the mind designed better than it is? British Journal of Medical Psychology, 71, 353-401.

Gilbert, P. (2000). Evolurionary theory and cognitive therapy. New York: Springer Publishing Company.

Gonçalves, D., H., \& Heldt, E. (2009). Transtorno da ansiedade na infância como preditor de psicopatologia em adultos. Revista Gaúcha de Enfermagem, 30(3), 533-541.

Hannesdottir, D. K., \& Ollendick, T. H. (2007). The role of emotion regulation in the treatment of child 
anxiety disorders. Clinical Child and Family Psychological Review, 10, 275-293.

Jacobs, M., Snow., J., Geraci, M., Vythilingam, M., Blair, R. J. B.., Charney, D., et al. (2008). Association between level of emotional intelligence and severity of anxiety in generalized social phobia. Journal of Anxiety Disorders, 22, 1487-1495.

Janeiro, A. C. (2013). Ansiedade aos exames/avaliações. Estudando e caracterizando este fenómeno no Ensino Básico e Secundário. Tese mestrado em Psicologia da Educação, especialidade em contextos educativos. Universidade dos Açores.

In-Albon, T., \& Schneider, S. (2007). Psychotherapy of childhood ansiety disorders: A meta-analysis. Psychotherapy and Psychosomatics, 76, 15-24. doi: 10.1159/000096361.

Layne, A. E.; Bernart, D. H.; Victor, A. M. \& Bernstein, G. A. (2008). Generalized anxiety disorder in a nonclinical sample of children: Symptom presentation and predictors of impairment. Journal of Anxiety Disorders, 23(2), 283-289.

Lizeretti, N., P., Extremeta, N., \& Rodríguez, A. (2012). Perceived Emotional Intelligence and Clinical Symptoms in Mental Disorders. Psychiatric Quarterly. 83, 407-418.

Manfro, G.G., Isolan, L., Blaya, C., Santos, L., \& Silva, M. (2002). Estudo retrospectivo da associação entre transtorno de pânico em adultos e transtorno de ansiedade na infância. Revista Brasileira de Psiquiatria, 24(2), 26-9.

Mara, D., \& Mara, E-L. (2010). Aspects concerning the manifestation of the students' emotional

Intelligence. Procedia Social and Behavioral Sciences, 5, 2379-2384.

Martins, A., C., Almeida, J., P., \& Viana, V. (2014). Ansiedade social na infância e pré-adolescêcia: Adaptação para o português de Portugal da SASC-R. Psicologia: Reflexão e crítica, 27(2), 300-307. doi: 10.1590/1678-7153.201427210.

Martins, A., Ramalho, N., \& Morin, E. (2010). A comprehensive meta-analysis of the relationship between emotional intelligence and health. Personality and Individual Differences, 49,554-564.

Mayer, J. D. (1997). What is Emotional Intelligence? In P. Salovey \& D. J. Sluyter (Eds.), Emotional Development and Emotional Intelligence (pp. 3-31). New York: Basic Books.

Mennin, S. D., Heimberg, R. G., Turk, C. L., \& Fresco, D. M. (2005). Preliminary evidence for an emotion dysregulation model of generalized anxiety disorder. Behaviour Research and Therapy, 43, 1281-1310.

Pina, A. A.; Silverman, W. K.; Alfano, C. A. \& Saavedra, L. M. (2002). Diagnostic efficiency of symptoms in the diagnosis of DSM-IV: generalized anxiety disorder in youth. Journal of Child Psychology and Psychiatry, 43 (7), 959-967.

Rosen, J. B., \& Schulkin, J. (1998). From normal fear to pathologiacl anxiety. Psychological Review, 105, 325-350.

Ruiz-Aranda, D., Salguero, J., \& Cabello, R. (2012). Can an Emotional Intelligence Program improve Adolescents' Psychosocial Adjustment? Results from the
Intemo Project. Social Behavior and Personality, 40(8), 1373-1380.

Salters-Pedneault, K., \& Diller, J., W. (2013). A preliminary study of anxiety, negative affect, experiential avoidance, and delaying of aversive events. Benhaviour Change. 4(30), 241-248.

Salters-Pedneault, K., Roemer, L., Tull, M. T., Rucker, L., \& Mennin, D. S. (2006). Evidence of broad deficits in emotion regulation associated with chronic worry and generalized anxiety disorder. Cognitive Therapy and Research, 30, 469-480.

Shamsy, M., Khoshli, A.K., \& Asadi, J. (2013). Correlation between Mothers' Emotional Intelligence and Anxiety Disorders in Preschool Students of Gorgan Preschools. Journal of Psychology \& Behavioural Sciences, 1(2), 70-77.

Suveg, C., \& Zeman, J. (2004). Emotion regulation in children with anxiety disorders. Journal of Clinical Child and Adolescent Psychology, 33, 750-759.

Turk, C. L., Heimberg, R. G., Luterek, J. A., Mennin, D. S., \& Fresco, D. (2005). Emotion dysregulation in generalized anxiety disorder: a comparison with social anxiety disorder. Cognitive Therapy and Research, 29, 89-106.

Vianna, R., R., A., B., Campos, A., A., \& Landeira-Fernandez, J. (2009). Transtorno de ansiedade na infância e adolescência: Uma revisão. Revista Brasileira de Terapias Cognitivas,1(5), 46-61.

Wilson, G. S., Pritchard, M. E., \& Revalee, B. (2005). Individual differences in adolescent health symptoms: The effect of gender and coping. Journal of Adolescence, 28, 369-379. 